\title{
ZYGOMYCOTA CITADOS PARA CHILE
}

\section{(Zygomycota cited in Chile)}

\author{
Oscar Martínez V. \& Eduardo Valenzuela F. \\ Instituto de Microbiología, Facultad de Ciencias, \\ Universidad Austral de Chile. Casilla 167, Valdivia, Chile.
}

Palabras claves: Taxonomía. Zygomycota, Glomeromycota, Chile.

Key word: Taxonomy, Zygomycota, Glomeromycota, Chile.

\section{RESUMEN}

Se hizo un estudio bibliográfico sobre taxa pertenecientes a las Divisiones Zygomycota y Glomeromycota, elaborandose un listado actualizado de los hongos pertenecientes a estos taxa citados para Chile, principalmente en revistas chilenas, hasta el año 2002. Los taxa fueron agrupadas de acuerdo a la taxonomía moderna citada por Kirk et al.,(2001). Para Chile actualmente se citarían 3 taxa pertenecientes a los Glomeromycota, 87 a los Zygomycota, de los cuales 81 pertenecen a los Zygomycetes y 6 a los Trycomycetes.

\section{INTRODUCCION}

De acuerdo a Kirk et al. (2001), los hongos per-tenecientes a la División Zygomycota han sido separados taxonómicamente en 2 Clases: Zygomycetes y Tricomy-cetes. Los primeros constan de10 Ordenes, 32 Fami-lias, 124 géneros, 83 sinónimos y 870 especies. Los segundos por 3 Ordenes, 6 Familias, 55 géneros, 22 sinónimos y 218 especies. La nueva División Glome-romycota (Shübler, et al. 2001), fue removida de la polifilética división Zigomycota donde el orden Glo-males de esta última, fue sustituido por Glomerales.

De las 1088 especies pertenecientes a los Zygomycota, sólo una pequeña fracción (90 taxa) han sido aisladas en Chile, un tercio de estas citas aparecen recopiladas en el texto Flora Fungosa Chilena de los autores Mújica \& Vergara (1945), obra que fue reactualizada en 1980 por Oehrens. Por otra parte, Lazo (1996), entrega un listado donde cita 31 especies de Zygomycota, de las cuales 27 coinciden con las previamente registradas por Mújica et $a l$. (1980). Con el correr del tiempo, se han aislado nuevas taxa de Zygomycota y esta información se encuentra dispersa, además, la obra de los autores antes señalados no ha sido reactualizada y algunas especies desde el punto de vista taxonómico o de la nomenclatura no corresponden a lo que inicialmente se describió, más aún, algunos taxa posteriores a 1980 citadas para Chile también presentan problemas taxonómicos.

\section{ABSTRACT}

A bibliographical study about taxa belonging to the Zygomycota and Glomeromycota Division was carried out. Based on the gathered information, an updated list of fungi included in the above taxonomic groups and cited in Chile mainly in chilean issues until 2002 was prepared. Taxa were grouped according to the modern taxonomy referred to by Kirk et al. (2001). As a result, there are in Chile 3 taxa belonging to the Glomeromycota, 87 belonging to the Zygomycota, 81 to the Zygomycetes, and 6 to the Trycomycetes .

El objetivo de este trabajo es proporcionar una lista actualizada de los taxa pertenecientes a las Divisiones Zygo y Glomeromycota citadas (en revistas preferen-temente nacionales) para Chile hasta el año 2002.

\section{MATERIALES Y METODOS}

La información acerca de los taxa citados para Chile, se recopiló inicialmente según lo citado por Mújica et al. (1980), posteriormente por una revisión de revistas científicas del área Micológica y afines (Ej. Bol. Micol., Rev. Chil. Historia Nat., Arch. Med. Veter., Agric. Téc., Mycological Research, Diccinario de los Hongos (Kirk et al.,2001), etc.). Además, se revisaron bases de datos (CAB, EBSCOhost Web Publishing, The CABI Bioscien-ce, CBS Database of Fungal Names).

Se elaboraron tablas comparativas de los taxa citados por Mújica et al. (1980), con la nomenclatura y taxonomía actual.

\section{RESULTADOS}

En Tabla 1, primera columna se indican los taxa según Kirk et al. (2001), en la segunda los citadas por Mújica et al. (1980) y en la última los citados para Chile entre 1977-2002, según la bibliografía consultada. 
Tabla 1. Glomeromycota y Zygomycota citados para Chile

Taxa según Schubler et al. y Citados en Mújica et al.(1980) Nuevas Taxa citadas para

Kirk et al. (2001)

Glomeromycota Schubler et al., phylum novo (2001) Mycol. Res. 105:

1413-1421 Anteriormente incluido en

Zygomycota

Glomerales

Glomeraceae (= Glomaceae)

Glomus

Glomus sp. (nativos) (citado por: Rubio et al., 1994)

Glomus acaulospora (citado por: González et al., 1995)

Endogone fuegiana Speg.

Glomus etunicatum (citado por: Borie Endogone reniformis Bres. et al., 1996)

\section{Zygomycota}

Endogonales

Endogonoceace

Endogone

Entomophthorales

Ancylistaceae

Conidiobolus

Entomophthoraceae

Empusa (= Entomophthora)

Entomophthora
Endogone flammicorona (citada por: Garrido, 1986)

Empusa muscae (Fr.) Cohn.

Entomophthora aphidis Hoffman Entomophthora apiculata (Thaxt.)

Entomophthora grylli Fres.

Entomophthora muscae (Cohn) Fres Conidiobolus apiculatus (Thaxter) Entomophthora parvispora Mac-leod Re-maudiere \& Séller 1980 (citado por: \& Carl Aruta et al., 1984)

Entomophthora oehrensiana Aruta, Carrillo \& Montealegre

Conidiobolus major (Thaxter) Remaudiere y Séller (citado por: Aruta \& Carrillo, 1989)

Endogone lactiflua (citada por: Garrido, 1986)
Ento

Entomophthora Sphaerosperma

Fres.

Entomophthora thaxteriana (Petch) Hall \& Bell .

(n)


Continuación Tabla 1

Erynia

Strongwellsea

Neozygitaceae

Neozygites

\section{Cunninghamella sp.}

\section{Mucorales}

Chaetocladiaceae

Chaetocladium

Cunninghamellaceae

Cunninghamella
Erynia (Zoophthora) occidentalis (Thax-ter) Humber y Ben Ze'Ev. (citado por: Aruta \& Carrillo, 1989)

Erynia (Zoophthora) humbersi sp. nov. (cit. por: Aruta \& Carrillo, 1989)

Strongwellsea oehrensiana n. sp. (citado por: Aruta et al., 1984)

Neozygites parvispora (Mac Leod, Tirrel and Carl) Remaudiere \& Keller (citado por: Aruta et al., 1984)

Neozygites lageniformis (Thaxter) Ramaudiere y Keller 1980 (citado por: Aruta \& Carrillo, 1989)

Chaetocladium brefeldii $\mathrm{v}$. Tieghem \& Lemond. (citado por Piontelli et al., 1981) Parásito facultativo de $\mathrm{Mu}$ corales, cosmopolita.

Cunninghamella echinulata var. antarctica (Caretta \& Piontelli) R.Y. Zheng \& C.G. Chen. comb.nov = Cunninghamella antarctica Caretta \& Piont. (citado por: Caretta \& Piontelli, 1977)

Cunninghamella elegans Lendner (citado por: Musalem et al., 1984)

Cunninghamella bainieri Naumov. (citado por: Carias, 1997; Valenzuela et al., 2000; Olavarria, 2000)

Gilbertellaceae

Gilbertella

Mortierellaceae

Micromucor
Gilbertella persicaria (Hedí) Hesseltine (citado por: Toro et al., 1993)

Micromucor ramannianus var angulisporus Naumov ex Vánová (= Mortierella ramanniana var. angulispora ( Naumov ) Linnem.) (citado por: Anabalón, 2000; Martínez et al., 2001)

Micromucor isabellinus (Oudem.) von Arx (=Mortierella isabellina Oudem.) (citado por: Carias, 1997; Olavarria, 2000)

Micromucor ramannianus var .ramannianus (A. Moller) von Arx (= Mortierella ramanniana var. autotrophica E. H. Evans) (citado por: Martínez et al., 2001) 
Continuación Tabla 1

Rhizomucor

Rhizopus

Zygorhynchus

Pilobolaceae

Pilobulos

Syncephalastraceae

Syncephalastrum

Género incierto

Blastocystis *

Thamnidiaceae

Backusella

Thamnostylum

Zoopagales

Helicocefaphalidaceae

Rhopalomyces

Piptocephalidaceae

Piptocephalis
Mucor heterosporum Fischer Mucor hygrophilus Oud. Mucor mucedo (L.) Fres. Mucor pirelloides Lendt. Mucor racemosus Fres. Mucor stolonifer Ehr.

Rhizopus nigricans Ehrb. Rhizopus oryzae (Fr.) Lind. Rhizopus stolonifer (Fr.) Lind.

Pilobolus crystallinus (Wigg.) Tode

Syncephalastrum sp.
Mucor fragilis Wehmer (cit.por: Piontelli et al., 1984)

Mucor racemosus f. spaerosporus (Hagen) Schipper (=Mucor globosus Fischer) (citado por: Piontelli \& Grixolli, 1993)

Mucor piriformis Fischer (cit. por: Latorre, 1995)

Rhizomucor spp. (cit. por:García et al., 2001; Zaror et al., 1999)

Rhizomucor miehei (Cooney \& Emerson) Schipper (cit. por: Piontelli \& Toro, 1983)

Rhizomucor pusillus (Linolt) Schipper (citado por: Piontelli \& Toro, 1983; Piontelli \& Grixolli, 1993)

Rhizopus pseudochinensis Yamazaki (citado por: Piontelli \& Toro, 1983)

Rhizopus microsporus v. Tiegh. var. chinensis (Saito) Schipper \& Stalpers (citado por: Piontelli et al., 1984)

Zygorhynchus moelleri Will. (cit. por: Piontelli \& Grixolli, 1993; Kunstmann et al., 1986; Carias, 1997; Olavarria, 2000)

Zygorhinchus heterogamus (citado por: Santibáñez, 2000)

Pilobolus kleinii v. Thieghem (citado por Piontelli et al., 1998)

Syncephalastrum racemosus Cohn. (Piontelli, comunicación personal, parásito facultativo de Mucorales, cosmopolita, aislados de cereales)

Blastocystis hominis (citado por: Mercado \& Arias, 1991)

Backusella lamprospora (Lendn.) Benny \& R.K. Benj (= Mucor lamprosporus Lendner) (citado por: Piontelli et al., 1984; 1986)

Thamnostylum piriforme (Bain.) von Arx \& Upadh.(cit. por Piontelli et al., 1981)

Rhopalomyces elegans Corda (citado por Piontelli et al., 1998)

Piptocephalis lepidula (March.) Benjam. (citado por Piontelli et al., 1981)

(*) Actualmente clasificado como Stramenopiles (Heterokonta) perteneciente al reino Chromista 
Continuación Tabla 1

Umbelopsis

Mucoraceae

Absidia

Actinomucor

Apophysomyces

Circinella

Gongronella

Mucor (=Mucedo)

Amylomyces

Mucor
Umbelopsis vinacea (Dixon-Stew.) von. Arx (= Mortierella vinacea (Möller) Linnem.) (citado por: Carias, 1997; Anabalón, 2000; Martínez et al., 2001; Olavarria, 2000; Santibáñez, 2000)

Umbelopsis nana (Linnem.) von Arx (=Mortierella nana Linnem.) (citado por Carias, 1997; Anabalón, 2000; Santibáñez, 2000; Valenzuela et al., 2001)

Absidia sp.(cit.por:García et al.,2001) $\quad$ Absidia corymbifera (Cohn) Saccardo \& Trotter (cit. por: Piontelli \& Toro, 1983; Barrera, 1997);

Absidia glauca Hagem (cit. por: Musalem et al., 1984; Carias, 1997),

Absidia cylindrospora Hagem (cit. por: Musalem et al., 1984; Carias, 1997; Olavarria, 2000; Santibáñez, 2000)

Absidia spinosa Lendner (cit. por: Carias, 1997; Olavarria, 2000)

Actinomucor repens Schosta-ko- Actinomucor elegans (Eidom) Benj. \& Hesselt. vitsch (=Actinomucor elegans (Ei- (citado por Piontelli et al., 1981)

dam) Benj. \& Hesselt.)

Apophysomyces spp. (citado por: García et al., 2001)

Circinella sp.

Circinella mucoroides Saito (citado por: Piontelli et al., 1984)

Circinella muscae (Sorokine) Berlese \& De Toni (citado por: Piontelli et al., 1984)

Gongronella butleri (Lendner) Peyronel \& Dal Vesco (citado por: Carias, 1997; Olavarria, 2000; Santibáñez, 2000)

Mucedo violacens sp.

Amylomyces rouxii Calmette( = Mucor rouxianus (Calmette) Wehmer (citado por: Piontelli et al., 1984)

Mucor sp.

Mucor aquosus Mart

Mucor circenelloides $\mathrm{v}$. T.

Mucor corymbosus Wallr

Mucor debaryanus Schost.

Mucor glomerula (Bainer) Lend- Piontelli et al., 1996)Mucor plumbeus Bonord. ner
Mucor hiemalis Wehmer (cit. por: Musalem et al., 1984; Piontelli et al., 1981; 1984; 1986; Piontelli \& Grixolli, 1993; Carias, 1997; Barrera, 1997; Olavarria, 2000; Anabalón, 2000)

Mucor hiemalis Wehmer f. hiemalis (cit. por: (cit. por: Piontelli \& Grixolli, 1993; Zaror et al., 1999)

Mucor abundans Povah (cit. por: Piontelli et al., 1981; 1984) 
En la Tabla 2, primera columna se indican los taxa según Kirck et al. (2001) y en la segunda columna los taxa citadas para Chile entre 1980-2002 de acuerdo a las fuentes bibliográficas consultadas.

Tabla 2. Trichomycetes citados para Chile

\begin{tabular}{|c|c|}
\hline Taxa según Kirk et al (2001) & $\begin{array}{c}\text { Nuevas Taxa citadas para Chile } \\
(1980-2002)\end{array}$ \\
\hline $\begin{array}{l}\text { Amoebidiales } \\
\text { Amoebidiaceae } \\
\text { Paramoebidium } \\
\text { Harpellales } \\
\text { Harpellaceae } \\
\text { Harpella } \\
\text { Stachylina } \\
\text { Legeriomycetaceae } \\
\text { Smittium }\end{array}$ & $\begin{array}{l}\text { Paramoebidium grande Lichtw. \& Arenas (citado por: Lichtwardt \& Arenas, } \\
\text { 1996) } \\
\text { Harpella meridionalis Lichtw. \& Arenas (citado por: Lichtwardt \& Arenas, 1996) } \\
\text { Stachylina ceratopogonidarum Lichtw. \& Arenas (citado por: Lichtwardt \& } \\
\text { Arenas, 1996) } \\
\text { Smittium cylindrosporum Lichtw. \& Arenas (citado por: Lichtwardt \& Arenas, } \\
\text { 1996) } \\
\text { Smittium imitatum Lichtw. \& Arenas (citado por: Lichtwardt \& Arenas, 1996) } \\
\text { Smittium simulatum Lichtw. \& Arenas (citado por: Lichtwardt \& Arenas, 1996) }\end{array}$ \\
\hline
\end{tabular}

\section{DISCUSION}

De acuerdo a Kirk et al. (2001), a nivel mundial se han registrado 1088 especies de hongos pertenecientes a los Zygomycota. Según Mújica et al. (1980), para Chile han sido citados 33 taxa pertenecientes a esta División, la mayoría de ellas (20) pertenecen al Orden Mucorales, posteriormente Lazo (1996), citó 31 especies de Zygomycota, omitiendo Entomophthora aphidis, Circinella sp., Mucor sp., Syncephalastrum sp., Cunninghamella sp., Mucedo violacens y citando en su lugar a Cunninghamella antarctica, Rhizopus pseudochinensis, Rhizomucor pusillus y $R$. miehei. De acuerdo a la información recolectada a partir de 1977 al 2002 se registran para Chile 51 taxa de Zygomycetes y 6 para Trycomycetes.

De acuerdo a CABI Bioscience Database (2003), algunos de los nombres registrados por Mújica et al. (1980), han variado. En otros casos se conserva el nombre, pero los autores de las especies han sido modificados como se indican: ej.Empusa muscae E. Cohn (1855), cuyo nombre actual corresponde a Entomophthora muscae (E. Cohn) G. Winter., Entomophthora grylli Fresen. (1856), nombre actual Entomophaga grylli (Fresen.) Batko, (1964). Entomophthora parvispora D.M. Macleod \& K.P. Carl, perteneciente a la Familia Entomophthoraceae, cuyo nombre actual es Neozygites parvispora (D.M. Macleod \& K.P. Carl) Remaud. \& S. Keller (1980), perteneciente a la Familia Neozygitaceae. Mucor glomerula Lendn y Actinomucor repens Schostak., corresponden a la misma especie cuyo nom- bre actual es Actinomucor elegans (Eidam) C.R. Benj. \& Hesselt.

Mucor pirelloides Lendn., perteneciente a la Familia Mucoraceae, cuyo nombre actual es Pirella circinans Bainier, (1882) y pertenece a la Familia Thamnidiaceae. Las 3 taxa siguientes: Mucor stolonifer Ehrenb., Rhizopus nigricans Ehrenb y Rhizopus stolonifer (Ehrenb.) Vuill., corresponden hoy taxonómicamente a Rhizopus stolonifer var. stolonifer (Ehrenb.) Vuill. (1902).

También algunos nombres de taxa citados entre el período 1980 a 2002 han variado, según CABI Bioscience Database (2003), como se indican: Erynia (Zoophthora) occidentalis (Thaxter) Humber y Ben Ze'Ev., cuyo nombre actual es Erynia occidentalis (Thaxt.) Humber \& Ben Ze'Ev.

Erynia (Zoophthora) humbersi sp. nov., cuyo nombre actual es Erynia humberi Aruta \& Carrillo (1989), por último Strongwellsea oehrensiana n. sp., cuyo nombre actual es Strongwellsea oehrensiana Aruta, Carrillo \& Monteal (1984). Algunos taxa pertenecientes a Mortierella han presentado modificaciones taxonómicas, a saber, Mortierella ramanniana var. angulispora (Naumov) Linnem (1941), cuyo nombre actual es Micromucor ramannianus var. angulisporus Naumov ex Vánová, (1991). Mortierella ramanniana var. autotrophica E.H. Evans (1971), cuyo nombre actual es Micromucor ramannianus var. ramannianus (A. Møller) Arx, (1982). Mortierella vinacea Dixon-Stew. (1932), nombre actual Umbelopsis vinacea (DixonStew.) Arx, (1982). Mortierella nana Linnem. (1941), 
parte de la Familia Mucoraceae.

Otro grupo que presenta modificaciones taxonómicas es Mucor: Mucor lamprosporus Lendn. (1907) perteneciente a la Familia Mucoraceae cuyo nombre actual es Backusella lamprospora (Lendn.) Benny \& R.K. Benj. (1975), pasa a pertenecer a la Familia Thamnidiaceae. $\mathbf{M u}$ cor rouxianus (Calmette) Wehmer (1900) nombre actual Amylomyces rouxii Calmette, (1976). Mucor globosus A. Fisch. (1892) nombre actual Mucor racemosus f. sphaerosporus (Hagem) Schipper, (1970). Por último, Rhizopus pseudochinensis M. Yamaz. (1918) pasa a denominarse Rhizopus oryzae Went \& Prins. Geerl., (1895) y Absidia cylindrospora Hagem (1908) pasa a llamarse Absidia cylindrospora var. cylindrospora Hagem, (1908).

Finalmente, como se muestra en la Tabla 2, es importante hacer notar que solo hasta el trabajo de Lichtwardt \& Arenas (1996) se citan hongos pertene-cientes a la Clase Trichomycetes para Chile, y además todos ellos corresponden a especies nuevas para la ciencia.

Debe destacarse que el listado de los Zigomycota citados para Chile, posiblemente sólo sea parcial, debido a que no incluye trabajos de autores extranjeros que recolectaron material diverso en Chile y publicados en revistas científicas internacionales. Además hay importantes grupos de taxa que no registra la Tabla 1 debido a que se han detectado para el país en trabajos rutinarios sin ser publicados.

\section{AGRADECIMIENTOS}

Al Proyecto DID 200210 de la Universidad Austral de Chile. Al Profesor Dr. Eduardo Piontelli, Universidad de Valparaíso. Por sus valiosos comentarios y correcciones del manuscrito.

\section{REFERENCIAS}

Anabalón, L. (2000). Determinación enzimática cualitativo y de la capacidad biodegradativa de cepas de hongos aisladas de hojas de Nothofagus pumilio (OPEP. Et endl) Krasser. Tesis Lic. en Ciencias Biológicas. Universidad Austral de Chile. Facultad de ciencias.

Aruta, C., Carrillo, R. \& Montealegre, J. (1984). Determinación para Chile de hongos del orden Entomophthorales (Zygomycetes). Agro Sur 12: $36-42$

Aruta, C. \& Carrillo, R. (1989). Identificación de hongos del orden Entomophthorales en Chile. III. Agro Sur 17: 10-18

Barrera, S. (1997). Estudio taxonómico y enzimático cualitativo de los hongos Deuromycotina y Zigomycotina aislados de aserrín de Pinus radiata $\mathrm{D}$. Don almacenado a la intemperie en el aserradero Vista Alegre de Valdivia. Tesis Lic. en Ciencias Biológicas. Universidad Austral de Chile. Facultad de ciencias.

Borie , F.; Rubio, R.; Moraga, E. \& Morales, A. (1996). Efecto de la roca fosfórica sobre la doble simbiosis de hongo micorrizógenos-VA y Rhizobium trifolii en Trébol Rosado. Agricultura Técnica (Chile) $56: 237-243$

CABI Bioscience Databases (2003). The CABI Bioscience and CBS Database of Fungal Names. <http://www.indexfungorum.org/Names/ Names.asp $>$.

Caretta, G. \& Piontelli, E. (1977). Microsporum magellanicum and Cunninghamella antarctica, new species isolated from Australic and Antarctic soil of Chile. Sabouraudia 15:1-10

Carias, P. (1997). Biocaracterización micológica de un suelo sometido a tres manejos distintos. Tesis Magíster en ciencias mención Protección Vegetal. Universidad Austral de Chile. Facultad de ciencia Agrarias.

García, P.; Beltrán, C.; Guzmán, A.; León, P.; Arredondo, M.; Fonseca, esculentum Mill.) y su incidencia sobre el desarrollo del cultivo. Agricultura Técnica 54:7-14Garrido, N. (1986). Survey of ectomycorrhizal fungi associated with exotic forest trees in Chile. Nova Hedwigia 43:423-442

González, B.; Godoy R. \& Figueroa, H. (1995). Dinámica estacional de los hongos micorrícicos vesículo-arbusculares en ecosistemas dunarios del centro-sur de Chile. Agricultura Técnica, 55:267-272
Hawksworth, D., Kirk, P., Sutton, B. \& Pegler, D. (1995). Dictionary of the fungi. $8^{\text {th }}$ Edition CAB International, Cambridge.

Kirk, P.M.; Cannon, J.C.; David, J.C. \& Stalpers, J.A. (2001). Dictionary of the fungi. $9^{\text {th }}$ Edition CAB Bioscience. CAB International, Cambridge

Kunstmann, E.; Osorio M. \& Peredo, H. (1986). Identificaciones micológicas en viveros forestales de la X Región de Chile. Bosque 7:51-56

Latorre, B. (1995). Enfermedades de las plantas cultivadas. Cuarta edición. Ediciones Universidad Católica de Chile.

Lazo, W. (1996). Especies fúngicas de Chile: índice alfabético. Boletín Micológico 11: 103-126

Lichtwardt, R. \& Arenas, J. (1996). Trichomycetes in aquatic insects from southern Chile. Mycologia 88: 844-857

Martínez, A.; Chiocchio, V. \& Godeas, A. (2001). Hyphomycetes celulolíticos en suelos de bosques de Nothofagus, tierra del fuego. Gayana Botánica 58:123-132

Mercado, R. \& Arias, B. (1991). Blastocystis hominis: Frecuencia de infección en pacientes ambulatorios del sector norte de Santiago, Chile. Boletín Chileno de Parasitología 46:30-32

Mújica, F. \& Vergara, C. (1945). Flora fungosa chilena. $1^{\text {a }}$ edición. Ministerio de agricultura, Dirección General Agricultura. Imprenta Stanley, Santiago, Chile.

Mújica, F.; Vergara, C. \& Oehrens, E. (1980). Flora fungosa chilena. $2^{\mathrm{a}}$ edición. Revisada y actualizada por Edgar Oehrens B. Universidad de Chile, Facultad de Agronomía. Ciencias Agrícolas n ${ }^{\circ} 5$. 
Musalem, S.; Steiner, W. \& Contreras, O. (1984). Producción de celulasa de hongos aislados de madera y suelos del sur de Chile. Boletín Micológico 2: 17-25

Olavarria, G. (2000). Caracterización enzimática cualitativa de cepas fúngicas de un suelo trumao y determinación mediante parámetros químicos de su capacidad para biodegradar paja de trigo. Tesis Lic. en Agronomía. Universidad Austral de Chile. Facultad de Agronomía.

Piontelli, E.; Toro, M., \& Caretta, G. (1981). Cophrophilous fungi of the horses. Mycopathologia 74:89-105

Piontelli, E. \& Toro, M. (1983). Hongos termofilos en nidos de pájaros. Boletín Micológico 1:157-176

Piontelli, E.; Toro, M. \& Casanova, D. (1984). Diversity-Dominance and succession of fungal communities in sandy soils (a beach of $\mathrm{V}$ Región-Chile) on keratinic substrata. Boletín Micológico 2:73 - 89

Piontelli, E.; Toro, M., \& Casanova, D. (1986). Microcomunidades fúngicas en zonas altiplánica chilena. Estudios sobre substratos queratinicos . I. Rev. Arg. Micología 9:26-32

Piontelli, E. \& Grixolli, M. (1993). Microhongos del suelo asociados a pastos silvestres senescentes en una localidad precordillerana de la $9^{\text {a }}$ Región (Chile). Boletín Micológico 8: 3 - 11

Piontelli, E.; Grixolli, M. \& Moraga, S. (1996). Prospección micológica en rizosfera y rizoplano en un vivero forestal de Eucalyptus globulus Labill. en la V Región - Chile. Boletín Micológico 11:17-32

Rubio, R.; Uribe, R.; Borie, F.; Moraga, E.; Contreras, A. (1994). Micorrizas vesículo-arbusculares (VA) en horticultura. Velocidad de infección en lechuga (Lactuca sativa L.) y tomate (Lycorpesicon esculentum Mill.) y su incidencia sobre el desarrollo del cultivo. Agricultura Técnica 54:7-14

Santibáñez, C. (2000). Taxonomía y determinación enzimática cuali- tativa de microhongos aislados de sedimentos del lago Riñihue, siete tributarios y efluentes. Tesis Lic. En Biología Marina. Universidad Austral de Chile. Facultad de ciencias, Escuela Biología Marina.

Schüßler, A.; Schwarzott, D. \& Walker, C. (2001). A new fungal phylum, the Glomeromycota: phylogeny and evolution. Mycol. Res. 105:1413-1421

Toro, M.; Díaz, A. \& Piazze, F. (1993). Microhongos filamentosos y levaduriformes asociados a pimienta negra (Piper nigrum L.). Boletín Micológico 8:77 - 83

Valenzuela, E.; Carias, P.; Pinochet, D. \& Polette, M. (2000). Nuevas citas de hongos aislados de suelo trumao sometido a diferentes manejos agro-forestales en la X Región de Chile. Boletín. Micológico 15:1-134

Valenzuela, E.; Leiva, S. \& Godoy, R. (2001). Variación estacional y potencial enzimático de microhongos asociados con la descomposición de hojarasca de Nothofagus pumilio. Rev. Chilena Historia Natural 74: 737-749

Zaror, L.; Araya, O. \& Varela, C. (1999). Determinación de hongos y bacterias termofílicas en heno y paja en criaderos de caballos criollos chilenos. Archivos Medicina Veterinaria 31:119-124 